\title{
Quinolone resistance: much more than predicted
}

\author{
Alvaro Hernández ${ }^{1,2}$, María B. Sánchez ${ }^{1,2}$ and José L. Martínez ${ }^{1,2 *}$ \\ Departamento de Biotecnología Microbiana, Centro Nacional de Biotecnología, CSIC, Madrid, Spain \\ 2 Consorcio de Investigación Biomédica de Epidemiología y Salud Pública, Barcelona, Spain
}

\section{Edited by:}

David W. Graham, Newcastle

University, UK

Reviewed by:

Charles Knapp, University of

Strathclyde, UK

Timothy Lapara, University of

Minnesota, USA

Vivian Miao, University of British

Columbia, Canada

Amy Pruden, Virginia Tech, USA

*Correspondence:

José L. Martínez, Departamento de

Biotecnología Microbiana, Centro

Nacional de Biotecnología (CSIC),

Darwin 3, Campus Universidad

Autónoma de Madrid, Cantoblanco,

28049 Madrid, Spain.

e-mail:jImtnez@cnb.csic.es
Since quinolones are synthetic antibiotics, it was predicted that mutations in target genes would be the only mechanism through which resistance could be acquired, because there will not be quinolone-resistance genes in nature. Contrary to this prediction, a variety of elements ranging from efflux pumps, target-protecting proteins, and even quinolone-modifying enzymes have been shown to contribute to quinolone resistance. The finding of some of these elements in plasmids indicates that quinolone resistance can be transferable. As a result, there has been a developing interest on the reservoirs for quinolone-resistance genes and on the potential risks associated with the use of these antibiotics in non-clinical environments. As a matter of fact, plasmid-encoded, quinolone-resistance qnr genes originated in the chromosome of aquatic bacteria. Thus the use of quinolones in fish-farming might constitute a risk for the emergence of resistance. Failure to predict the development of quinolone resistance reinforces the need of taking into consideration the wide plasticity of biological systems for future predictions. This plasticity allows pathogens to deal with toxic compounds, including those with a synthetic origin as quinolones.

Keywords: quinolone resistance, qnr, MDR efflux pump, quinolone inactivation, transfer of quinolone resistance
Quinolones are a group of synthetic antibiotics with great clinical relevance. The first quinolones were mainly used for treating Gramnegative bacterial infections. However they were later on modified in order to become effective agents for treating both Gram-negative and Gram-positive infections. Among the quinolones derivatives, fluoroquinolones are a subgroup of widely used quinolones, which structure presents a nitrogen instead of a carbon at position 8 of their naphthyridine nucleus and a fluorine substitution at position 6 (Ball, 2000). These synthetic antimicrobials interact with the bacterial topoisomerases DNA gyrase and topoisomerase IV (Shen et al., 1989; Khodursky and Cozzarelli, 1998). Both enzymes are composed of two pairs of subunits and participate together in DNA replication, transcription, recombination, and repair. Binding of quinolones to these targets arrests DNA replication fork progression and consequently leads to cell death.

It was proposed early that mutations in genes encoding for target proteins (Crumplin and Odell, 1987) or for transporters (Piddock, 1999) would be the only mechanisms through which quinolone resistance could be achieved. This proposal was based on available information concerning the origins of antibiotic resistance genes, which bacterial pathogens have acquired after the introduction of antibiotics for the treatment of infections. It has been described that the families of plasmids present in human pathogens before and after the medical use of antibiotics are the same, the only difference being that the latter harbor resistance genes (Datta and Hughes, 1983). In agreement with these findings, it was proposed that resistance determinants acquired by bacterial pathogens through horizontal gene transfer (HGT) should originate in non-pathogenic bacteria. Most antibiotics are produced by environmental microorganisms (Waksman and Woodruff, 1940), which need to harbor resistance determinants in order to avoid the activity of self-produced antimicrobials. Because of this, it has been widely accepted that antibiotic producers are the origin of resistance genes acquired through HGT by human pathogens (Benveniste and Davies, 1973; Webb and Davies, 1993; Davies, 1994). The identification of a large number of resistance determinants in soil-dwelling actinomycetes, which are the main family of antibiotic producers, reinforced this view (D'Costa et al., 2006; Wright, 2007). Based on these hypotheses it was proposed that environmental bacteria would not carry quinolone-resistance genes because there are no quinolone producers and hence no microorganisms would be confronted with the selective pressure that these antimicrobials might exert. Since the origin of resistance genes acquired by human pathogens through HGT is the environmental microbiota, the predictions were that acquisition of quinolone-resistance genes through HGT would be unfeasible. The aim of this article is to review the current quinolone-resistance mechanisms disseminated among human pathogens in the frame of these former predictions. We also address the consequences that the discovery of HGT-acquired quinolone resistance may have on the utilization of these antimicrobials. Finally, we discuss the reasons why these predictions failed in the aim of developing more accurate methodologies for predicting the emergence of resistance in bacterial pathogens (Martinez et al., 2007).

For a long time and in agreement with what was originally anticipated, the only reported quinolone-resistance mechanisms were mutations in target genes. Even nowadays, mutations in topoisomerases remain the most prevalent mechanism among bacterial populations for the acquisition of high-level resistance to these antimicrobials (Jacoby, 2005). In Gram-negative bacteria, highlevel quinolone resistance is mainly due to mutations on the genes encoding for the gyrase subunits, gyrA and $g y r B$ (mainly in $g y r A$ ), whereas mutations in parC and parE, which encode for the subunits 
of topoisomerase IV, are secondary. The reason for this finding is that in Gram-negatives, bacterial topoisomerase IV is less sensitive to quinolones than gyrase, therefore it constitutes a secondary target for this family of antibiotics (Drlica and Zhao, 1997). Indeed, mutations in par genes are only found in Gram-negative bacteria that also harbor gyrA mutations (Hopkins et al., 2005). The opposite happens for Gram-positive organisms. In these bacterial species, topoisomerase IV is usually the primary target of quinolones (Ferrero et al., 1994). Consequently, quinolone-resistance mutations occur first in parC in Gram-positive bacteria.

The characterization of multidrug (MDR) efflux pumps, which are encoded in the core genomes of all bacterial species (Nikaido, 1998; Saier et al., 1998; Alonso et al., 1999; Piddock, 2006a), opened new avenues for understanding the mechanisms involved in quinolone resistance. Notably, despite a synthetic origin, quinolones are among the most common substrates of these pumps (Hooper, 1999). In agreement with this information, it was shown that MDR pumps indeed contribute to the resistance to quinolones (Cohen et al., 1989). These results showed that, opposite to early predictions, more elements besides target mutations might be involved in the development of quinolone resistance. Chromosomally encoded MDR efflux pumps are usually expressed at very low level as the consequence of the activity of specific transcriptional regulators (usually repressors; Grkovic et al., 2002). This low-level expression is enough to allow MDR pumps to contribute to intrinsic resistance to quinolones (Li et al., 1994; Vila and Martinez, 2008). In addition, mutants presenting de-repressed high-level expression of MDR efflux pumps are selected in clinical settings by antimicrobial therapy. As a results of the overexpression of MDR pumps, these mutants are less susceptible to quinolones than wild-type strains (acquired resistance; Cohen et al., 1989; Ziha-Zarifi et al., 1999; Jalal et al., 2000; Alonso and Martinez, 2001).

Overexpression of MDR efflux pumps does not account for high-level resistance to quinolones and usually results in a small increase in minimal inhibitory concentrations (MICs). There are some exceptions however, such as SmeDEF from Stenotrophomonas maltophilia (Alonso and Martinez, 1997, 2000). In this organism, high-level resistance can be achieved by mutants that overexpress this efflux pump. In addition, the combined overexpression of different efflux pumps in the same microorganism, can further increase the level of quinolone resistance (Yang et al., 2003). Finally, different studies have shown that the highest level of resistance to quinolones is achieved when both mechanisms, mutations in the target genes and efflux through MDR systems, take place at the same time (Llanes et al., 2006). Altogether these results indicate that the contribution of mechanisms that reduce the quinolones intracellular concentrations for developing high-level resistance to quinolones should not be underestimated. One relevant aspect of MDR efflux pumps is their wide range of substrates (Paulsen, 2003 ), in such a way that their overexpression results in a phenotype of cross-resistance to several antibiotics. This means that quinolone resistance can be achieved upon selective pressure with a non-quinolone antibiotic (second order selection) when both drugs are substrates of the same MDR efflux pump (Cohen et al., 1989). This is a new concept, useful for understanding the selective forces and the mechanisms involved in the acquisition of quinolone resistance by bacterial pathogens.
To date, efflux pumps responsible for the extrusion of quinolones have been described in Gram-positive and Gram-negative bacteria. It has been demonstrated that transporters belonging to four of the five families of multidrug resistance (MDR) systems; the ATP binding cassette (ABC) family, the major facilitator superfamily (MFS), the resistance-nodulation division (RND) family, and the multidrug and toxic compound extrusion (MATE) family, are capable of extruding quinolones (Poole, 2000a,b). In most cases, quinolones are pumped out by MDR systems with relative low substrate specificity that confer low-level quinolone resistance when overexpressed. Only in a few cases, such as NorC in Staphylococcus aureus (Truong-Bolduc et al., 2006) or Rv1634 in Mycobacterium tuberculosis (De Rossi et al., 2002), pump specificity for quinolones has been demonstrated, and given the synthetic nature of these antimicrobials the primary role of these efflux pumps should be other than extrusion of fluoroquinolones. Indeed, several studies have shown that MDR efflux pumps might contribute to different aspects in bacterial physiology, including extrusion of virulence factors, cell-signal trafficking, and detoxification of toxic cellular metabolites (Piddock, 2006b; Martinez et al., 2009b).

Even after the discovery that mechanisms other than mutations in target genes contribute to quinolone resistance, the general consensus remained that resistance determinants in plasmids or transposons should not be expected and hence the contribution of HGT to quinolone resistance must be negligible. Once again, this prediction was based on the notion that environmental bacteria do not need to carry quinolone-resistance elements because of the synthetic origin of these drugs. In spite of these predictions, the possibility of plasmid-encoded quinolone resistance was demonstrated in vitro (Gomez-Gomez et al., 1997; Martinez et al., 1998). Later on, the first plasmid-mediated quinolone resistance, was described. This finding indicated that the acquisition of quinolone resistance by HGT is possible. The plasmid-encoded gene responsible for the observed resistance phenotype was called $q n r$ (Martinez-Martinez et al., 1998). Qnr belongs to the pentapeptide repeat protein (PRP) family (Vetting et al., 2006), and protects bacterial topoisomerases (DNA gyrase an topoisomerase IV) from the activity of quinolones activity. At the moment of writing this article, $5 q n r$ families, $q n r A$, qnrB, qnrS, qnrC, and $q n r D$ (Tran and Jacoby, 2002; Hata et al., 2005; Jacoby et al., 2006; Strahilevitz et al., 2007; Cavaco et al., 2009; Wang et al., 2009) have been described in plasmids disseminated among bacterial pathogens. The presence of these genes in the chromosomes of environmental bacteria has been reported as well (Arsene and Leclercq, 2007; Rodriguez-Martinez et al., 2008; Sanchez et al., 2008), and it has been proposed that these bacterial species might constitute the environmental reservoir for quinolone-resistance genes. For instance, Shewanella algae is the origin of plasmid-encoded qnrA genes (Poirel et al., 2005b) and different Vibrionaceae species might be the reservoir for other plasmid-encoded qnr genes (Poirel et al., 2005a; Cattoir et al., 2007). While it is clear that plasmid-encoded qnr genes contribute to acquired resistance to quinolones, the role that these determinants might play on intrinsic resistance when they are chromosomally encoded, has been explored in less detail. One exception is S. maltophilia Smqnr, which has been shown to contribute to intrinsic quinolone resistance in this bacterial species (Sanchez and Martinez, 2010). In addition to Qnr determinants other members 
of the PRP family, might be relevant for quinolone resistance. This is the case of MfpA that contributes to the intrinsic resistance of Mycobacterium to these antimicrobials (Montero et al., 2001). In spite of their contribution to intrinsic resistance, it is unlikely that this is the natural function of these genes given the synthetic origin of quinolones. Some hypotheses about their putative functional role have been proposed. Since $q n r$ binds DNA gyrase (Tran and Jacoby, 2002; Tran et al., 2005; Jacoby et al., 2006), which controls the DNA supercoiling and therefore global bacterial transcription, Qnr might modulate the expression of several genes through its interaction with DNA gyrase, in response to environment changes. It has been also proposed that Qnr proteins might protect DNA gyrase against some toxins, in a similar way as that described for another member of the PRP family, McbG, which protects bacteria from the activity of microcin B17 (Heddle et al., 2001).

An important particularity of plasmid-encoded $q n r$ genes is that, although they have been found in a large variety of plasmids (Strahilevitz et al., 2009), the DNA sequences (genetic environment) surrounding $q n r$ genes are rather similar. The conserved synteny found around plasmid-encoded $q n r$ genes suggests that there have been a limited number of acquisition events of such genes from their original bacterial host. Indeed, the analysis of the genetic environments surrounding qnrA or qnrB genes shows that both are usually integrated in complex sull-type integrons and associated with ISCR1 (Nordmann and Poirel, 2005; Garnier et al., 2006). In addition to $q n r$, these integrons usually harbor other antibiotic resistance genes, such as $\beta$-lactamases or aminoglycoside inactivating enzymes (Wang et al., 2003). The qnrS genes are not harbored by integrons, however they are frequently associated with Tn 3 transposon, which contains a TEM-1 $\beta$-lactamase gene (Hata et al., 2005; Strahilevitz et al., 2009). Finally, the plasmids themselves might contain other resistance determinants, being particularly relevant the association of qnr genes with genes coding for extended spectrum $\beta$-lactamases and AmpC $\beta$-lactamases (Strahilevitz et al., 2009). These associations with other resistance determinants have likely favored the dissemination and maintenance of $q n r$ genes even under situations in which quinolones are not used for therapy, since the additional resistance genes will allow for co-selection of $q n r$.

The discovery of plasmid-encoded quinolone-resistance genes dismantled the idea that quinolone resistance could never be achieved by means of HGT. This has profound implications, because mutation-acquired resistance can only disseminate through clonal expansion (Martinez and Baquero, 2000), whereas HGT allows dissemination of resistance genes among different strains and different bacterial species (Baquero et al., 2009; Martinez et al., 2009a). The next surprise came with the demonstration that even mutation-acquired resistance to quinolones might be transferred through HGT. It was found that chromosomal parC and gyrA mutations could be transferred by transformation along with the quinolone-resistance phenotype in clinical isolates of Streptococcus pneumoniae (Ferrandiz et al., 2000). Furthermore, transfer of this mutation-acquired resistance from the viridans group streptococci to Streptococcus pneumoniae has been described (Balsalobre et al., 2003), indicating that commensal bacteria can be a source of target-mutation-based quinolone resistance.
In the last years additional elements conferring resistance to quinolones have been described indicating that we are still far away from getting a comprehensive understanding of the mechanisms leading to this phenotype. Among these new mechanisms, two plasmid-encoded quinolones efflux pumps, named QepA (Yamane et al., 2007) and OqxAB (Hansen et al., 2004, 2007) have been described. QepA was the first described plasmid-encoded quinolone efflux transporter. Because of its similarities to the MFS family of MDR pumps of actinomycetes it has been proposed that the protein originated in these Gram-positive bacteria (Yamane et al., 2007) and was transferred afterward to Enterobacteriaceae plasmids, where now it can disseminate. In addition to quinolones, this determinant can efflux a narrow range of substrates, including erythromycin, ethidium bromide, and acriflavine. Other antibiotics that are common substrates of other MDR efflux pumps are not extruded by this pump. In the case of OqxAB, the acquisition of a plasmid encoding oq $x A B$ entails, in addition to an increase in resistance to quinolones, an increase in the bacterial resistance to a wider range of antibiotics such as tetracycline or choramphenicol and also an increased resistance to biocides like benzalkonium chloride or triclosan, since all these compounds are substrates of the pump (Hansen et al., 2007).

The last frontier to be explored on the mechanisms of quinolone resistance was the possibility that quinolone-inactivating enzymes might be present in bacterial populations. At a first sight, the degradation of quinolones would be problematic since bacterial populations did not evolved in ecosystems containing these substrates and consequently they should not harbor degradation pathways for these compounds. This is particularly relevant for fluoroquinolones, given that halogenated compounds are among the most biodegradation-resilient molecules. On the other hand there is a general consensus that several antibiotic-modifying enzymes (not degradative ones) evolved mainly in producers either as antibioticdetoxifying elements, or as enzymes involved in the antibiotics biosynthetic pathways (Benveniste and Davies, 1973; Webb and Davies, 1993; Davies, 1994, 1997). As rightly stated by Courvalin (1990), the producing organism of quinolone is Homo sapiens, and it does not require to harbor in its genome a gene encoding for a quinoloneinactivating enzyme. Other antibiotic-modifying enzymes have a primary function in the bacterial metabolism and their capability for modifying the antibiotic is a consequence of the structural similarities between the antimicrobial and the original substrate of the enzyme. This is the case of some aminoglycosides or $\beta$-lactams, which resemble some of the intermediates of peptidoglycan bricks (Payie et al., 1995; Macinga and Rather, 1999). Given the synthetic origin of quinolones and the lack of structural analogs of these antibiotics among bacterial metabolic intermediates, the finding of a quinolone-inactivating enzyme came as a surprise. This enzyme, encoded by the $a a c\left(6^{\prime}\right)-I b-c r$ gene, appeared as a modification of an original aminoglycoside acetyltransferase, which has acquired the ability to inactivate quinolones (ciprofloxacin and norfloxacin) by $\mathrm{N}$-acetylation the amino nitrogen on its piperazinyl group. This novel activity of the enzyme is the consequence of two amino acids changes (Trp102Arg and Asp179Tyr; Robicsek et al., 2006). The fact that these modifications somehow compromise the enzymatic activity against aminoglycosides (Robicsek et al., 2006) indicates that the evolved enzyme has been likely selected by quinolones during 
antimicrobial treatment. Recently, the association of the $\operatorname{aac}\left(6^{\prime}\right)$ $I b$-cr gene with genes encoding for the $\beta$-lactamase CTXM- 15 or extended spectrum $\beta$-lactamases has been reported (Coque et al., 2008; Pitout et al., 2008). In the same way as plasmid-encoded $q n r$ determinants, these associations allow for co-selection of resistance even in the absence of quinolones.

So far in this article, we have described several determinants that might contribute to the development of quinolone resistance in bacterial pathogens. Expression of most of these novel elements confers low-level antimicrobial resistance, while high-level resistance requires mutations in the target genes. One exception to this rule is S. maltophilia (Sanchez et al., 2009), which quinoloneresistant mutants do not seem to present mutations in the genes coding for the quinolones targets (Ribera et al., 2002; Valdezate et al., 2005). S. maltophilia harbors in its chromosome a qnr gene (Sanchez et al., 2008; Shimizu et al., 2008) and the genes encoding for the SmeDEF efflux pump, which is very efficient at extruding quinolones (Alonso and Martinez, 2000). These features might be relevant for the development of quinolone resistance in this bacterial pathogen.

In spite of the low-level resistance derived from these mechanisms, their role in quinolone resistance should not be underestimated. As mentioned above, the level of resistance in clinical isolates is frequently achieved by a combination of different mechanisms. Furthermore, it has been described that the presence of qnr genes increases the mutant prevention concentration and thus favors selection of quinolone-resistant mutants, including those harboring mutations in target genes (Martinez-Martinez et al., 1998; Rodriguez-Martinez et al., 2007). A final consideration regards the possibility of selecting quinolone resistance in the absence of quinolones. Efflux pumps can extrude different antibiotics besides quinolones. Thus the presence of any of these antibiotics will coselect quinolone resistance (cross-resistance). The same applies for plasmid-encoded quinolone-resistance elements, because the plasmids usually harbor other resistance genes, and selection with any of the antibiotics for which the plasmid confers resistance will select for quinolone resistance as well (co-resistance).

Niels Bohr once quoted that "Predictions can be very difficult especially about the future". The history about predicting quinolone resistance is a very good example of this issue. This is not to say that we should not try to predict the possible emergence of resistance before it appears (Martinez et al., 2007), but rather that we cannot discard potential mechanisms using ab initio hypotheses, that might seem realistic but are not comprehensive enough. In the case of quinolone resistance, the predictions failed because the plasticity (and in occasions the lack of specificity) of biological systems was inadequately considered. In contrast to the idea that each resistance determinant has been developed in bacterial populations in order to avoid the activity of a specific antibiotic, it has been recently discussed that the situation is much more complex (Fajardo et al., 2009; Martinez et al., 2009a). In addition to well known specific resistance mechanisms (c.a. $\beta$-lactamases), a large number of

\section{REFERENCES}

Alonso, A., and Martinez, J. L. (1997). Multiple antibiotic resistance in Stenotrophomonas maltophilia. Antimicrob. Agents Chemother. 41, 1140-1142.
Alonso, A., and Martinez, J. L. (2000). Cloning and characterization of SmeDEF, a novel multidrug efflux pump from Stenotrophomonas maltophilia. Antimicrob. Agents Chemother. 44, 3079-3086.

proteins, with an original function unrelated to antibiotic resistance, might also contribute to this phenotype (Fajardo et al., 2008; Tamae et al., 2008; Alvarez-Ortega et al., 2010). The term "intrinsic resistome" (Fajardo et al., 2008) was coined from these studies in order to refer to the group of chromosomally encoded elements, not acquired by HGT, that contribute to the basal level of resistance to a given antibiotic that is common to all members of a bacterial species. This description is independent of the clinical definition of resistance, which is based in MICs breakpoints. In the case of quinolones, recent publications indicate that several determinants contribute to both intrinsic and acquired resistance to these drugs despite their synthetic origin (Breidenstein et al., 2008).

The finding that quinolone resistance is a transferable trait changes the focus to the elements relevant for the dissemination of resistance to such drugs. If just mutation-driven resistance were observed, the only relevant risk for human health should be the use of quinolones for human therapy, given that the only available source of resistance would be the human pathogens themselves. The use of these drugs for animal farming might be controversial because some bacterial clones might infect both humans and farm animals, but these risks should be minor in a scenario in which resistance is not transferable. In fact, quinolones have been largely used in fish-farming without major concerns about the associated risks for human health until recently (Cabello, 2006; Baquero et al., 2008). Once transfer of quinolone resistance was demonstrated, it became clear that the utilization of these drugs might be risky for human health even if they were used for processes other than treating human infections, given that the barriers for gene transfer among different bacterial species can be overcome if the selective pressure is strong enough (Baquero et al., 2008). The intense antibiotic selective pressure, not only in the clinical environment, but also in farming and aquaculture (Cabello, 2006; Baquero et al., 2008; Martinez, 2008, 2009), is likely favoring the persistence of quinolone-resistant bacterial populations and the transmission of their resistance elements. For instance, it has been reported that the same broad host range IncU-type plasmid containing a qnrS2 gene was found in two Aeromonas isolates from two different nonconnected geographical locations, the Seine river in France and the Lugano lake at Switzerland (Cattoir et al., 2008; Picao et al., 2008). This integration of qnrgenes in plasmids of water-dwelling bacteria might be the first step toward their transfer to human pathogens (Martinez, 2008). The fact that $q n r$ genes are originally found in bacteria with an aquatic habitat, further supports the relevance of natural ecosystems in the development of quinolone resistance, despite the synthetic origin of these drugs.

\section{ACKNOWLEDGMENTS}

Work in our laboratory is supported by grants BIO2008-00090 from the Spanish Ministry of Science and Innovation, and KBBE227258 (BIOHYPO) and HEALTH-F3-2010-241476 (PAR). Thanks are given to Dr. Alvarez-Ortega for English correction and to Dr. Willem van Schaik for making us aware of Bohr's sentence.

Alonso, A., and Martinez, J. L. (2001). Expression of multidrug efflux pump SmeDEF by clinical isolates of Stenotrophomonas maltophilia. Antimicrob. Agents Chemother. 45, 1879-1881.
Alonso, A., Rojo, F., and Martinez, J. L. (1999). Environmental and clinical isolates of Pseudomonas aeruginosa show pathogenic and biodegradative properties irrespective of their origin. Environ. Microbiol. 1, 421-430. 
Alvarez-Ortega, C., Wiegand, I., Olivares, J., Hancock, R. E., and Martinez, J. L. (2010). Genetic determinants involved in the susceptibility of Pseudomonas aeruginosa to beta-lactam antibiotics. Antimicrob. Agents Chemother. 54, 4159-4167.

Arsene, S., and Leclercq, R. (2007). Role of a $q n r$-like gene in the intrinsic resistance of Enterococcus faecalis to fluoroquinolones. Antimicrob. Agents Chemother. 51, 3254-3258.

Ball, P. (2000). Quinolone generations: natural history or natural selection? J. Antimicrob. Chemother. 46(Suppl. T1), 17-24.

Balsalobre, L., Ferrandiz, M. J., Linares, J., Tubau, F., and de la Campa, A. G. (2003). Viridans group streptococci are donors in horizontal transfer of topoisomerase IV genes to Streptococcus pneumoniae. Antimicrob. Agents Chemother. 47, 2072-2081.

Baquero, F., Alvarez-Ortega, C., and Martinez, J. L. (2009). Ecology and evolution of antibiotic resistance. Environ. Microbiol. Rep. 1, 469-476.

Baquero, F., Martinez, J. L., and Canton, R. (2008). Antibiotics and antibiotic resistance in water environments. Curr. Opin. Biotechnol. 19, 260-265.

Benveniste, R., and Davies, J. (1973). Aminoglycoside antibiotic-inactivating enzymes in actinomycetes similar to those present in clinical isolates of antibiotic-resistant bacteria. Proc. Natl. Acad. Sci. U.S.A. 70, 2276-2280.

Breidenstein, E. B., Khaira, B. K., Wiegand, I., Overhage, J., and Hancock, R. E. (2008). Complex ciprofloxacin resistome revealed by screening a Pseudomonas aeruginosa mutant library for altered susceptibility. Antimicrob. Agents Chemother. 52, 4486-4491.

Cabello, F. C. (2006). Heavy use of prophylactic antibiotics in aquaculture: a growing problem for human and animal health and for the environment. Environ. Microbiol. 8, 1137-1144.

Cattoir, V., Poirel, L., Aubert, C., Soussy, C. J., and Nordmann, P. (2008). Unexpected occurrence of plasmidmediated quinolone resistance determinants in environmental Aeromonas spp. Emerging Infect. Dis. 14, 231-237.

Cattoir, V., Poirel, L., Mazel, D., Soussy, C. J., and Nordmann, P. (2007). Vibrio splendidus as the source of plasmidmediated QnrS-like quinolone resistance determinants. Antimicrob. Agents Chemother. 51, 2650-2651.

Cavaco, L. M., Hasman, H., Xia, S., and Aarestrup, F. M. (2009). qnrD, a novel gene conferring transferable quinolone resistance in Salmonella enterica serovar Kentucky and Bovismorbificans strains of human origin. Antimicrob. Agents Chemother. 53, 603-608.
Cohen, S. P., McMurry, L. M., Hooper, D. C., Wolfson, J.S., and Levy, S. B. (1989). Cross-resistance to fluoroquinolones in multiple-antibiotic-resistant (Mar) Escherichia coli selected by tetracycline or chloramphenicol: decreased drug accumulation associated with membrane changes in addition to OmpF reduction. Antimicrob. Agents Chemother. 33, 1318-1325.

Coque, T. M., Novais, A., Carattoli, A., Poirel, L., Pitout, J., Peixe, L., Baquero, F., Canton, R., and Nordmann, P. (2008). Dissemination of clonally related Escherichia coli strains expressing extended-spectrum betalactamase CTX-M-15. Emerging Infect. Dis. 14, 195-200.

Courvalin, P. (1990). Plasmid-mediated 4-quinolone resistance: a real or apparent absence? Antimicrob. Agents Chemother. 34, 681-684.

Crumplin, G. C., and Odell, M. (1987). Development of resistance to ofloxacin. Drugs 34(Suppl. 1), 1-8.

D'Costa, V. M., McGrann, K. M., Hughes, D. W., and Wright, G. D. (2006). Sampling the antibiotic resistome. Science 311, 374-377.

Datta,N., and Hughes,V.M.(1983).Plasmids of the same Inc groups in Enterobacteria before and after the medical use of antibiotics. Nature 306, 616-617.

Davies, J. (1994). Inactivation of antibiotics and the dissemination of resistance genes. Science 264, 375-382.

Davies, J. E. (1997). Origins, acquisition and dissemination of antibiotic resistance determinants. Ciba Found. Symp. 207, 15-27.

De Rossi, E., Arrigo, P., Bellinzoni, M., Silva, P. A., Martin, C., Ainsa, J. A., Guglierame, P., and Riccardi, G. (2002). The multidrug transporters belonging to major facilitator superfamily in Mycobacterium tuberculosis. Mol. Med. 8, 714-724.

Drlica, K., and Zhao, X. (1997). DNA gyrase, topoisomerase IV, and the 4-quinolones. Microbiol. Mol. Biol. Rev. 61, 377-392.

Fajardo, A., Linares, J. F., and Martinez, J. L. (2009). Towards an ecological approach to antibiotics and antibiotic resistance genes. Clin. Microbiol. Infect. 15(Suppl. 1), 14-16.

Fajardo, A., Martinez-Martin, N., Mercadillo, M., Galan, J. C., Ghysels, B., Matthijs, S., Cornelis, P., Wiehlmann, L., Tummler, B., Baquero, F., and Martinez, J. L. (2008). The neglected intrinsic resistome of bacterial pathogens. PLoS ONE3, e1619. doi: 10.1371/ journal.pone.0001619

Ferrandiz, M. J., Fenoll, A., Linares, J., and De La Campa,A. G. (2000). Horizontal transfer of parC and gyrA in fluoroquinolone-resistant clinical isolates of Streptococcus pneumoniae. Antimicrob. Agents Chemother. 44, 840-847.
Ferrero, L., Cameron, B., Manse, B., Lagneaux, D., Crouzet, J., Famechon, A., and Blanche, F. (1994). Cloning and primary structure of Staphylococcus aureus DNA topoisomerase IV: a primary target of fluoroquinolones. $\mathrm{Mol}$. Microbiol. 13, 641-653.

Garnier, F., Raked, N., Gassama, A., Denis, F., and Ploy, M. C. (2006) Genetic environment of quinolone resistance gene qnrB2 in a complex sull-type integron in the newly described Salmonella enterica serovar Keurmassar. Antimicrob. Agents Chemother. 50, 3200-3202.

Gomez-Gomez,J.M.,Blazquez,J.,Espinosa De Los Monteros, L. E., Baquero, M. R. Baquero, F., and Martinez, J. L. (1997). In vitro plasmid-encoded resistance to quinolones. FEMS Microbiol. Lett. 154 271-276.

Grkovic, S., Brown, M. H., and Skurray, R. A. (2002). Regulation of bacterial drug export systems. Microbiol. Mol. Biol. Rev. 66, 671-701.

Hansen, L. H., Jensen, L. B., Sorensen, H. I., and Sorensen, S. J. (2007). Substrate specificity of the OqxAB multidrug resistance pump in Escherichia coliand selected enteric bacteria. J. Antimicrob. Chemother. 60, 145-147.

Hansen, L. H., Johannesen, E., Burmolle, M., Sorensen, A. H., and Sorensen, S. J. (2004). Plasmid-encoded multidrug efflux pump conferring resistance to olaquindox in Escherichia coli. Antimicrob. Agents Chemother. 48, 3332-3337.

Hata, M., Suzuki, M., Matsumoto, M. Takahashi, M., Sato, K., Ibe, S., and Sakae, K. (2005). Cloning of a novel gene for quinolone resistance from a transferable plasmid in Shigella flexneri 2b. Antimicrob. Agents Chemother. 49, 801-803.

Heddle, J. G., Blance, S. J., Zamble, D. B., Hollfelder, F., Miller, D. A., Wentzell, L. M., Walsh, C. T., and Maxwell, A. (2001). The antibiotic microcin B17 is a DNA gyrase poison: characterisation of the mode of inhibition. J. Mol. Biol. 307, 1223-1234.

Hooper, D. C. (1999). Mechanisms of fluoroquinolone resistance. Drug Resist. Updat. 2, 38-55.

Hopkins, K. L., Davies, R. H., and Threlfall, E. J. (2005). Mechanisms of quinolone resistance in Escherichia coli and Salmonella: recent developments. Int. J. Antimicrob. Agents 25, 358-373.

Jacoby, G.A. (2005). Mechanisms of resistance to quinolones. Clin. Infect. Dis. 41(Suppl. 2), S120-S126.

Jacoby, G. A., Walsh, K. E., Mills, D. M. Walker, V. J., Oh, H., Robicsek, A., and Hooper, D. C. (2006). qnrB, another plasmid-mediated gene for quinolone resistance. Antimicrob. Agents Chemother. 50, 1178-1182.
Jalal, S., Ciofu, O., Hoiby, N., Gotoh, N., and Wretlind, B. (2000). Molecular mechanisms of fluoroquinolone resistance in Pseudomonas aeruginosa isolates from cystic fibrosis patients. Antimicrob. Agents Chemother. 44, 710-712.

Khodursky, A. B., and Cozzarelli, N. R. (1998). The mechanism of inhibition of topoisomerase IV by quinolone antibacterials. J. Biol. Chem. 273, 27668-27677.

Li, X. Z., Livermore, D. M., and Nikaido, H. (1994). Role of efflux pump(s) in intrinsic resistance of Pseudomonas aeruginosa: resistance to tetracycline, chloramphenicol, and norfloxacin. Antimicrob. Agents Chemother. 38, 1732-1741.

Llanes, C., Neuwirth, C., El Garch, F. Hocquet, D., and Plesiat, P. (2006). Genetic analysis of a multiresistant strain of Pseudomonas aeruginosa producing PER-1 beta-lactamase. Clin. Microbiol. Infect. 12, 270-278.

Macinga, D. R., and Rather, P. N. (1999). The chromosomal 2'-Nacetyltransferase of Providencia stuartii: physiological functions and genetic regulation. Front. Biosci. 4, D132-D140.

Martinez, J. L. (2008). Antibiotics and antibiotic resistance genes in natural environments. Science 321, 365-367.

Martinez, J. L. (2009). The role of natural environments in the evolution of resistance traits in pathogenic bacteria. Proc. R. Soc. B Biol. Sci. 276, 2521-2530.

Martinez, J. L., Alonso, A., GomezGomez, J. M., and Baquero, F. (1998). Quinolone resistance by mutations in chromosomal gyrase genes. Just the tip of the iceberg? J. Antimicrob. Chemother. 42, 683-688.

Martinez, J. L., and Baquero, F. (2000). Mutation frequencies and antibiotic resistance. Antimicrob. Agents Chemother. 44, 1771-1777.

Martinez, J.L., Baquero, F., and Andersson, D. I. (2007). Predicting antibiotic resistance. Nat. Rev. Microbiol. 5 , 958-965.

Martinez, J. L., Fajardo, A., Garmendia, L., Hernandez, A., Linares, J. F., MartinezSolano, L., and Sanchez, M. B. (2009a). A global view of antibiotic resistance. FEMS Microbiol. Rev. 33, 44-65.

Martinez, J. L., Sanchez, M. B., Martinez-Solano, L., Hernandez, A., Garmendia, L., Fajardo, A., and Alvarez-Ortega, C. (2009b) Functional role of bacterial multidrug efflux pumps in microbial natural ecosystems. FEMS Microbiol. Rev. 33, 430-449.

Martinez-Martinez, L., Pascual, A., and Jacoby, G. A. (1998). Quinolone resistance from a transferable plasmid. Lancet 351, 797-799. 
Montero, C., Mateu, G., Rodriguez, R., and Takiff, H. (2001). Intrinsic resistance of Mycobacterium smegmatis to fluoroquinolones may be influenced by new pentapeptide protein MfpA. Antimicrob. Agents Chemother. 45, 3387-3392.

Nikaido, H. (1998). Antibiotic resistance caused by Gram-negative multidrug efflux pumps. Clin. Infect. Dis. 27(Suppl. 1), S32-S41.

Nordmann, P., and Poirel, L. (2005). Emergence of plasmid-mediated resistance to quinolones in Enterobacteriaceae. J. Antimicrob. Chemother. 56, 463-469.

Paulsen, I. T. (2003). Multidrug efflux pumps and resistance: regulation and evolution. Curr. Opin. Microbiol. 6, 446-451.

Payie, K. G., Rather, P. N., and Clarke, A. J. (1995). Contribution of gentamicin 2'-N-acetyltransferase to the $\mathrm{O}$ acetylation of peptidoglycan in Providencia stuartii. J. Bacteriol. 177, 4303-4310.

Picao, R. C., Poirel, L., Demarta, A., Silva, C. S., Corvaglia, A. R., Petrini, O., and Nordmann, P. (2008). Plasmidmediated quinolone resistance in Aeromonas allosaccharophila recovered from a Swiss lake. J. Antimicrob. Chemother. 62, 948-950.

Piddock, L. J. (1999). Mechanisms of fluoroquinolone resistance: an update 1994-1998. Drugs 58, 11-18.

Piddock, L. J. (2006a). Clinically relevant chromosomally encoded multidrug resistance efflux pumps in bacteria. Clin. Microbiol. Rev. 19, 382-402.

Piddock, L. J. (2006b). Multidrugresistance efflux pumps - not just for resistance. Nat. Rev. Microbiol. 4 , 629-636.

Pitout, J. D., Wei, Y., Church, D. L., and Gregson, D. B. (2008). Surveillance for plasmid-mediated quinolone resistance determinants in Enterobacteriaceae within the Calgary Health Region, Canada: the emergence of aac(6')-Ib-cr. J. Antimicrob. Chemother. 61, 999-1002.

Poirel, L., Liard, A., Rodriguez-Martinez, J. M., and Nordmann, P. (2005a). Vibrionaceae as a possible source of Qnr-like quinolone resistance determinants. J. Antimicrob. Chemother. 56, 1118-1121.

Poirel, L., Rodriguez-Martinez, J. M., Mammeri, H., Liard, A., and Nordmann, P. (2005b). Origin of plasmid-mediated quinolone resistance determinant QnrA. Antimicrob. Agents Chemother. 49, 3523-3525.

Poole, K. (2000a). Efflux-mediated resistance to fluoroquinolones in Gramnegative bacteria. Antimicrob. Agents Chemother. 44, 2233-2241.
Poole, K. (2000b). Efflux-mediated resistance to fluoroquinolones in Grampositive bacteria and the mycobacteria. Antimicrob. Agents Chemother. 44, 2595-2599.

Ribera, A., Domenech-Sanchez, A., Ruiz, J., Benedi, V. J., Jimenez de Anta, M. T., and Vila, J. (2002). Mutations in gyrA and parC QRDRs are not relevant for quinolone resistance in epidemiological unrelated Stenotrophomonas maltophilia clinical isolates. Microb. Drug Resist. 8, 245-251.

Robicsek, A., Strahilevitz, J., Jacoby, G. A., Macielag, M., Abbanat, D., Park, C. H., Bush, K., and Hooper, D. C. (2006). Fluoroquinolone-modifying enzyme: a new adaptation of a common aminoglycoside acetyltransferase. Nat. Med. 12, 83-88.

Rodriguez-Martinez, J. M., Velasco, C., Briales, A., Garcia, I., Conejo, M. C., and Pascual, A. (2008). Qnr-like pentapeptide repeat proteins in Grampositive bacteria. J. Antimicrob. Chemother. 61, 1240-1243.

Rodriguez-Martinez, J. M., Velasco, C., Garcia, I., Cano, M. E., MartinezMartinez, L., and Pascual, A. (2007). Mutant prevention concentrations of fluoroquinolones for Enterobacteriaceae expressing the plasmid-carried quinolone resistance determinant qnrAl. Antimicrob. Agents Chemother. 51, 2236-2239.

Saier, M. H., Jr., Paulsen, I. T., Sliwinski, M. K., Pao, S. S., Skurray, R. A., and Nikaido, H. (1998). Evolutionary origins of multidrug and drug-specific efflux pumps in bacteria. FASEB J. 12, 265-274.

Sanchez, M. B., Hernandez, A., and Martinez,J.L.(2009).Stenotrophomonas maltophilia drug resistance. Future Microbiol. 4, 655-660.

Sanchez, M. B., Hernandez, A., Rodriguez-Martinez, J.M., MartinezMartinez, L., and Martinez, J. L. (2008). Predictive analysis of transmissible quinolone resistance indicates Stenotrophomonas maltophilia as a potential source of a novel family of Qnr determinants. $B M C$ Microbiol. 8, 148. doi: 10.1186/14712180-8-148

Sanchez, M. B., and Martinez, J. L. (2010). SmQnr contributes to intrinsic resistance to quinolones in Stenotrophomonas maltophilia. Antimicrob. Agents Chemother. 54, 580-581.

Shen, L. L., Kohlbrenner, W. E., Weigl, D., and Baranowski, J. (1989). Mechanism of quinolone inhibition of DNA gyrase. Appearance of unique norfloxacin binding sites in enzymeDNA complexes. J. Biol. Chem. 264, 2973-2978.
Shimizu, K., Kikuchi, K., Sasaki, T., Takahashi, N., Ohtsuka, M., Ono, Y., and Hiramatsu, K. (2008). Smqnr, a new chromosome-carried quinolone resistance gene in Stenotrophomonas maltophilia. Antimicrob. Agents Chemother. 52, 3823-3825.

Strahilevitz, J., Engelstein, D., Adler, A., Temper, V., Moses, A. E., Block, C., and Robicsek, A. (2007). Changes in qnr prevalence and fluoroquinolone resistance in clinical isolates of Klebsiella pneumoniae and Enterobacterspp.collected from 1990 to 2005. Antimicrob. Agents Chemother. 51, 3001-3003.

Strahilevitz, J., Jacoby, G. A., Hooper, D. C., and Robicsek, A. (2009). Plasmidmediated quinolone resistance: $\mathrm{a} \mathrm{mul-}$ tifaceted threat. Clin. Microbiol. Rev 22, 664-689.

Tamae, C., Liu, A., Kim, K., Sitz, D., Hong, J., Becket, E., Bui, A., Solaimani, P., Tran, K. P., Yang, H., and Miller, J. H. (2008). Determination of antibiotic hypersensitivity among 4,000 single-gene-knockout mutants of Escherichia coli. J. Bacteriol. 190, 5981-5988.

Tran, J. H., and Jacoby, G. A. (2002). Mechanism of plasmid-mediated quinolone resistance. Proc. Natl. Acad. Sci. U.S.A. 99, 5638-5642.

Tran, J. H., Jacoby, G. A., and Hooper, D. C. (2005). Interaction of the plasmidencoded quinolone resistance protein Qnr with Escherichia coli DNA gyrase. Antimicrob. Agents Chemother. 49, 118-125.

Truong-Bolduc, Q. C., Strahilevitz, J., and Hooper, D. C. (2006). NorC, a new efflux pump regulated by MgrA of Staphylococcus aureus. Antimicrob. Agents Chemother. 50, 1104-1107.

Valdezate, S., Vindel, A., Saez-Nieto, J. A., Baquero, F., and Canton, R. (2005). Preservation of topoisomerase genetic sequences during in vivo and in vitro development of high-level resistance to ciprofloxacin in isogenic Stenotrophomonas maltophilia strains. J. Antimicrob. Chemother. 56, 220-223.

Vetting, M. W., Hegde, S. S., Fajardo, J. E., Fiser, A., Roderick, S. L., Takiff, H. E., and Blanchard, J. S. (2006). Pentapeptide repeat proteins. Biochemistry 45, 1-10.

Vila, J., and Martinez, J. L. (2008). Clinical impact of the over-expression of efflux pump in nonfermentative Gramnegative bacilli, development of efflux pump inhibitors. Curr. Drug Targets 9, 797-807.

Waksman, S. A., and Woodruff, H. B. (1940). The soil as a source of microorganisms antagonistic to diseaseproducing bacteria. J. Bacteriol. 40, 581-600.
Wang, M., Guo, Q., Xu, X., Wang, X., Ye, X., Wu, S., Hooper, D. C., and Wang, M. (2009). New plasmid-mediated quinolone resistance gene, $\mathrm{qnrC}$, found in a clinical isolate of Proteus mirabilis. Antimicrob. Agents Chemother. 53 , 1892-1897.

Wang, M., Tran, J. H., Jacoby, G.A., Zhang, Y., Wang, F., and Hooper, D. C. (2003). Plasmid-mediated quinolone resistance in clinical isolates of Escherichia colifrom Shanghai, China. Antimicrob. Agents Chemother. 47, 2242-2248.

Webb, V., and Davies, J. (1993). Antibiotic preparations contain DNA: a source of drug resistance genes? Antimicrob. Agents Chemother. 37, 2379-2384.

Wright, G. D. (2007). The antibiotic resistome: the nexus of chemical and genetic diversity. Nat. Rev. Microbiol. 5, 175-186.

Yamane, K., Wachino, J., Suzuki, S., Kimura, K., Shibata, N., Kato, H. Shibayama, K., Konda, T., and Arakawa, Y. (2007). New plasmidmediated fluoroquinolone efflux pump, QepA, found in an Escherichia coliclinical isolate. Antimicrob. Agents Chemother. 51, 3354-3360.

Yang, S., Clayton, S. R., and Zechiedrich, E. L. (2003). Relative contributions of the AcrAB, MdfA and NorE efflux pumps to quinolone resistance in Escherichia coli. J. Antimicrob. Chemother. 51, 545-556.

Ziha-Zarifi, I., Llanes, C., Kohler, T., Pechere, J. C., and Plesiat, P. (1999). In vivo emergence of multidrug-resistant mutants of Pseudomonas aeruginosa overexpressing the active efflux system MexA-MexB-OprM. Antimicrob. Agents Chemother. 43, 287-291.

Conflict of Interest Statement: The authors declare that the research was conducted in the absence of any commercial or financial relationships that could be construed as a potential conflict of interest.

Received: 28 October 2010; accepted: 31 January 2011; published online: 11 February 2011.

Citation: Hernández A, Sánchez MB and Martinez JL (2011) Quinolone resistance: much more than predicted. Front. Microbio. 2:22. doi: 10.3389/fmicb.2011.00022

This article was submitted to Frontiers in Antimicrobials, Resistance and Chemotherapy, a specialty of Frontiers in Microbiology.

Copyright (® 2011 Hernández, Sánchez and Martinez. This is an open-access article subject to an exclusive license agreement between the authors and Frontiers Media $S A$, which permits unrestricted use, distribution, and reproduction in any medium, provided the original authors and source are credited. 